\title{
THE LAW OF THE ITERATED LOGARITHM FOR ADDITIVE FUNCTIONALS OF MARKOV CHAINS
}

\author{
YU MIAO AND GUANGYU YANG
}

\begin{abstract}
In the paper, the law of the iterated logarithm for additive functionals of Markov chains is obtained under some weak conditions, which are weaker than the conditions of invariance principle of additive functionals of Markov chains in M. Maxwell and M. Woodroofe 7] (2000). The main technique is the martingale argument and the theory of fractional coboundaries.
\end{abstract}

\section{INTRODUCTION}

Let $X_{0}, X_{1}, X_{2}, \ldots$ denote an ergodic stationary Markov chain with values in a measurable space $(\mathcal{X}, \mathcal{B})$, transition function $Q$, and stationary initial distribution $\pi$. Further, let $L^{2}(\pi)$ denote the space of (equivalence classes of) square integrable functions $g: \mathcal{X} \rightarrow \mathbb{R}$, for which $\|g\|^{2}:=\int_{\mathcal{X}} g^{2} d \pi<\infty$, and let $L_{0}^{2}(\pi)$ denote the set of $g \in L^{2}(\pi)$ for which $\int_{\mathcal{X}} g d \pi=0$. Given $g \in L_{0}^{2}(\pi)$, for $n \geq 1$, let

$$
S_{n}=S_{n}(g):=\sum_{i=1}^{n} g\left(X_{i}\right) .
$$

To describe more detailedly the model of additive functionals of above Markov chain, let $Q$ denote both the conditional distribution of $X_{1}$ given $X_{0}$ and the operator defined by

$$
Q h(x)=\int_{\mathcal{X}} h(y) Q(x ; d y)
$$

for a.e. $x \in \mathcal{X}$ and all $h \in L^{2}(\pi)$. It is easy to see that $Q$ is a contraction. Let

$$
V_{n} h=\sum_{k=0}^{n-1} Q^{k} h
$$

for $h \in L^{2}(\pi)$ and $n \geq 1$. Then each $V_{n}$ is a bounded linear operator. It is obviously that $V_{n} g(x)=\mathbb{E}\left[S_{n}(g) \mid X_{1}=x\right]$ for a.e. $x(\pi)$. For model (1.1), M. Maxwell and M.

Date: December 26, 2005.

2000 Mathematics Subject Classification. 60F05.

Key words and phrases. Markov chains, the law of the iterated logarithm, additive functionals, Dunford-Schwartz operator, fractional coboundaries, shift processes. 
Woodroofe [7] (2000) gave the central limit theorems under the following conditions:

$$
g \in L_{0}^{2}(\pi), \quad \sum_{n=1}^{\infty} n^{-3 / 2}\left\|V_{n} g\right\|<\infty,
$$

where $\|\cdot\|$ denotes the norm in $L^{2}(\pi)$.

It is well known that the law of the iterated logarithm (in short LIL) is closely related to the central limit theorem (in short CLT) in some sense. There are several approaches to these problems. If the chain is Harris recurrent, then the problems may be reduced to the independent case in a certain sense, see S.P. Meyn and R.L. Tweedie [8] (1993). If there is a solution to Poisson's equation, $h=g+Q h$, then the LIL and CLT problem may be reduced to the martingale case, see also M.I. Gordin and B.A. Lifsic [5] (1978) and S.P. Meyn and R.L. Tweedie [8] (1993). R.N. Bhattacharya [1] (1982) obtained CLT and LIL for ergodic stationary Markov process by discussion for infinitesimal generator. L.M. Wu [11] (1999) extended the forward-backward martingale decomposition of Meyer-Zheng-Lyons's type from the symmetric case to the general stationary situation and gave Strassen's strong invariance principle.

Our goal, in this paper, is to consider the problem that $S_{n}$ satisfies the LIL under some proper conditions. We will explore some extensions of the Poisson's equation approach, along the lines of C. Kipnis and S.R.S. Varadhan [6] (1986), and M. Maxwell and M. Woodroofe [7] (2000), to the cases where a solution is not required. We obtain the LIL for additive functionals of Markov chains in Section 2, where mainly depending on the LIL of martingale and the theory of fractional coboundaries developed by Y. Derriennic and M. Lin [3] (2001). In Section 3, we will give two examples, whose central limit theorems and invariance principles are discussed in M. Maxwell and M. Woodroofe [7] (2000), to illustrate our results.

\section{MAin Results}

For $\varepsilon>0$, let $h_{\varepsilon}$ be the solution to the equation $(1+\varepsilon) h=Q h+g$,

$$
h_{\varepsilon}=\sum_{n=1}^{\infty} \frac{Q^{n-1} g}{(1+\varepsilon)^{n}}=\varepsilon \sum_{n=1}^{\infty} \frac{V_{n} g}{(1+\varepsilon)^{n+1}} .
$$

Let $\pi_{1}$ be the joint distribution of $X_{0}$ and $X_{1}$, so that $\pi_{1}\left(d x_{0} ; d x_{1}\right)=Q\left(x_{0} ; d x_{1}\right) \times$ $\pi\left(d x_{0}\right)$; denote the norm in $L^{2}\left(\pi_{1}\right)$ by $\|\cdot\|_{1}$; and let

$$
H_{\varepsilon}\left(x_{0}, x_{1}\right)=h_{\varepsilon}\left(x_{1}\right)-Q h_{\varepsilon}\left(x_{0}\right)
$$

for $x_{0}, x_{1} \in \mathcal{X}$. Then for any $\varepsilon>0, H_{\varepsilon}$ is in $L^{2}\left(\pi_{1}\right)$; the norm of $H_{\varepsilon}$ is $\left\|H_{\varepsilon}\right\|_{1}^{2}=$ $\left\|h_{\varepsilon}\right\|^{2}-\left\|Q h_{\varepsilon}\right\|^{2}$; and $\int_{\mathcal{X}} H_{\varepsilon}\left(x_{0}, x_{1}\right) Q\left(x_{0} ; d x_{1}\right)=0$ for a.e. $x_{0}(\pi)$.

Now, let us give a few more definitions. For $\varepsilon>0$, let

$$
M_{n}(\varepsilon)=H_{\varepsilon}\left(X_{0}, X_{1}\right)+\cdots+H_{\varepsilon}\left(X_{n-1}, X_{n}\right)
$$

and

$$
R_{n}(\varepsilon)=Q h_{\varepsilon}\left(X_{0}\right)-Q h_{\varepsilon}\left(X_{n}\right)
$$


hence, by simple calculation,

$$
S_{n}(g)=M_{n}(\varepsilon)+\varepsilon S_{n}\left(h_{\varepsilon}\right)+R_{n}(\varepsilon) .
$$

For any fixed $\varepsilon, M_{n}(\varepsilon)$ is a square integrable martingale, where the martingale difference sequences is stationary ergodic.

Before our main results, we need mention the following lemmas.

Lemma 2.1. ( See M. Maxwell and M. Woodroofe [n] (2000))

: (R1) If (1.2) holds and $\left\|V_{n} g\right\|=O\left(n^{\alpha}\right)$ for some $\alpha>0$, then $\left\|h_{\varepsilon}\right\|=O\left(\varepsilon^{-\alpha}\right)$ as $\varepsilon \rightarrow 0$.

: (R2) For $0<\varepsilon, \delta<\infty,\left\|H_{\varepsilon}-H_{\delta}\right\|_{1}^{2} \leq(\varepsilon+\delta)\left[\left\|h_{\varepsilon}\right\|^{2}+\left\|h_{\delta}\right\|^{2}\right]$.

: (R3) If (1.2) holds, then $H=\lim _{\varepsilon \downarrow 0} H_{\varepsilon}$ exists in $L^{2}\left(\pi_{1}\right)$.

: (R4) If (1.2) holds, then

$$
S_{n}=M_{n}+R_{n}
$$

where $M_{1}, M_{2}, \ldots$ and $R_{1}, R_{2}, \ldots$ have strictly stationary increments, $M_{1}, M_{2}, \ldots$, is a square integrable martingale, and $\mathbb{E}\left(R_{n}^{2}\right)=o(n)$ as $n \rightarrow \infty$.

Recall that $T$ is a Dunford-Schwartz (DS) operator on $L^{1}$ of a probability space: if $T$ is a contraction of $L^{1}$ such that $\|T f\|_{\infty} \leq\|f\|_{\infty}$ for every $f \in L^{\infty}$. If $\theta$ is a measure preserving transformation in a probability space $(\Omega, \Sigma, \mu)$, then the operator $T f=f \circ \theta$ is a DS operator on $L^{1}(\mu)$. More generally, any Markov operator $\mathbb{P}$ with an invariant probability yields a positive DS operator.

Lemma 2.2. ( See Y. Derriennic and M. Lin [3] (2001))

: (L1) Let $T$ be a contraction in a Banach space $X$, and let $0<\beta<1$. If $\sup _{n}\left\|\frac{1}{n^{1-\beta}} \sum_{k=1}^{n} T^{k} y\right\|<\infty$, then $y \in(I-T)^{\alpha} X$ for every $0<\alpha<\beta$.

: (L2) Let $T$ is a DS operator in $L^{1}(\mu)$ of a probability space, and fix $1<p<\infty$, with dual $q=p /(p-1)$. Let $0<\alpha<1$, and $f \in(I-T)^{\alpha} L^{p}$. If $\alpha>1-\frac{1}{p}=\frac{1}{q}$, then $\frac{1}{n^{1 / p}} \sum_{k=0}^{n-1} T^{k} f \rightarrow 0$ a.e.

Lemma 2.3. ( See W.F. Stout [10] (1970)) Let $\left(Y_{i}, i \geq 1\right.$ ) be a stationary ergodic stochastic sequence with $\mathbb{E}\left[Y_{i} \mid Y_{1}, Y_{2}, \cdots, Y_{i-1}\right]=0$ a.e. for all $i \geq 2$ and $\mathbb{E} Y_{1}^{2}=1$. Then

$$
\limsup _{n \rightarrow \infty} \frac{\sum_{i=1}^{n} Y_{i}}{(2 n \log \log n)^{1 / 2}}=1, \quad \text { a.e. }
$$

Theorem 2.4. Let $g \in L_{0}^{2}(\pi)$ and $\left\|V_{n} g\right\|=O\left(n^{\alpha}\right)$ for some $\alpha<1 / 2$. Then, we have

$$
\limsup _{n \rightarrow \infty} \frac{S_{n}}{(2 n \log \log n)^{1 / 2}}=\|H\|_{1}, \text { a.e. }
$$

where $H$ is defined as Lemma 2.1 (R3). 
Proof. At first, we notice that, by Lemma $2.1(R 4), M_{n}$ is a square integrable martingale with strictly stationary increments and, by Lemma $2.3, M_{n}$ satisfies the law of the iterated logarithm, i.e.

$$
\limsup _{n \rightarrow \infty} \frac{M_{n}}{(2 n \log \log n)^{1 / 2}}=\|H\|_{1}, \text { a.e. }
$$

where $\|H\|_{1}$ is due to the ergodic theorem, i.e.

$$
\lim _{n \rightarrow \infty} \frac{1}{n} \sum_{i=1}^{n} H\left(X_{i-1}, X_{i}\right)^{2}=\|H\|_{1}^{2}, \text { a.e. }
$$

Next we will consider $R_{n}$. For each $n \geq 1$, let $\delta_{j}=2^{-j}, k_{n}$ be the unique integer $k$ for which $2^{k-1} \leq n<2^{k}$ and let $\varepsilon_{n}=2^{-k_{n}}$. Then, by (2.2) and Lemma 2.1 (R4), we have $R_{n}=M_{n}\left(\varepsilon_{n}\right)-M_{n}+\varepsilon_{n} S_{n}\left(h_{\varepsilon_{n}}\right)+R_{n}\left(\varepsilon_{n}\right)$ and, therefore,

$$
\begin{aligned}
\mathbb{E}\left(R_{n}^{2}\right) & \leq 4 \mathbb{E}\left[M_{n}\left(\varepsilon_{n}\right)-M_{n}\right]^{2}+4 \varepsilon_{n}^{2} \mathbb{E}\left[S_{n}\left(h_{\varepsilon_{n}}\right)\right]^{2}+4 \mathbb{E}\left[R_{n}\left(\varepsilon_{n}\right)\right]^{2} \\
& \leq 4 n\left\|H_{\varepsilon_{n}}-H\right\|_{1}^{2}+4\left\|h_{\varepsilon_{n}}\right\|^{2}+8\left\|h_{\varepsilon_{n}}\right\|^{2} .
\end{aligned}
$$

By Lemma 2.1 $(R 1),\left\|h_{\varepsilon_{n}}\right\|=O\left(n^{\alpha}\right)$ and by the definition of $\varepsilon_{n}$ and Lemma $2.1(R 2)$, there is a constant $C$ for which

$$
\left\|H_{\varepsilon_{n}}-H\right\|_{1} \leq \sum_{j=k_{n}+1}^{\infty}\left\|H_{\delta_{j}}-H_{\delta_{j-1}}\right\|_{1} \leq C \sum_{j=k_{n}+1}^{\infty} \delta_{j}^{1 / 2-\alpha}=O\left(n^{\alpha-1 / 2}\right) .
$$

Thus, we have $\mathbb{E}\left(R_{n}^{2}\right)=O\left(n^{2 \alpha}\right)$.

On $\mathcal{X} \times \mathcal{X}$, define $f\left(x_{0}, x_{1}\right)=g\left(x_{0}\right)-H\left(x_{0}, x_{1}\right)$, then

$$
R_{n}=S_{n}-M_{n}=\sum_{i=1}^{n-1}\left[g\left(X_{i}\right)-H\left(X_{i}, X_{i+1}\right)\right]=\sum_{i=1}^{n} f\left(X_{i}, X_{i+1}\right) .
$$

For sequence $x=\left(x_{i}\right)_{i \in \mathbb{N}} \in \mathcal{X}^{\mathbb{N}}$, define $F(x)=f\left(x_{0}, x_{1}\right)$ and let $\theta$ is the shift map on $\mathcal{X}^{\mathbb{N}}$ which is a contraction on the space $L^{2}\left(\pi_{1}\right)$. Then, we have

$$
F \in L^{2}\left(\pi_{1}\right) \text { and } R_{n}=\sum_{k=0}^{n-1} F \circ \theta^{k} .
$$

From the fact $\mathbb{E}\left(R_{n}^{2}\right)=O\left(n^{2 \alpha}\right)$, there exists a constant $1 / 2<\beta<1-\alpha$, such that

$$
\sup _{n}\left\|\frac{1}{n^{1-\beta}} \sum_{k=0}^{n-1} F \circ \theta^{k}\right\|<\infty \text {. }
$$

Since Lemma $2.2(L 1)$ and $\alpha<1 / 2$, we have $F \in(I-\theta)^{\eta} L^{2}\left(\pi_{1}\right)$, where $\eta \in(1 / 2,1-$ $\alpha$ ). By Lemma 2.2 (L2), we have

$$
\frac{1}{n^{1 / 2}} R_{n} \rightarrow 0, \quad \pi_{1}-\text { a.e. }
$$

Thus the result of the theorem holds by the fact, $S_{n}=M_{n}+R_{n}$. 
Corollary 2.5. If $g \in L^{2}(\pi)$, and either

$$
\sum_{k=1}^{\infty} k^{\delta-1 / 2}\left\|Q^{k} g\right\|<\infty
$$

or

$$
\sum_{k=1}^{\infty} k^{\delta}\left\|Q^{k} g\right\|^{2}<\infty
$$

for some $\delta>0$, then 2.4 holds.

Proof. By Kronecker's lemma and $V_{n} g=\sum_{k=0}^{n-1} Q^{k} g$, (2.11) means that

$$
n^{\delta-1 / 2}\left\|V_{n} g\right\| \leq n^{\delta-1 / 2} \sum_{k=1}^{n}\left\|Q^{k} g\right\| \rightarrow 0, \text { as } n \rightarrow \infty .
$$

It is obvious that, (2.12) implies (2.11). Form Theorem 2.4, the corollary holds.

Remarks 2.6. If $g \in L_{0}^{p}(\pi)$, for some $p>2$, then (2.11) or (2.12) are satisfied, M. Maxwell and M. Woodroofe [7] (2000) gave an invariance principle of

$$
\mathbb{B}_{n}(t)=\frac{1}{\sqrt{n}} S_{\lceil n t\rceil}, \quad 0 \leq t<1,
$$

and $\mathbb{B}_{n}(1)=\mathbb{B}_{n}(1-)$ for $n \geq 1$, where $\lceil x\rceil$ denotes the least integer that is greater than $x$.

\section{SOME APPLICATIONS}

In this section, we will give two examples to show that they satisfy the law of the iterated logarithm under some weakly dependent conditions. Here, the processes do not have recurrent points and are not strongly mixing. In fact, the two examples have been studied in M. Maxwell and M. Woodroofe [7] (2000) and we should thank their ideas and results.

EXAMPLE 1. ( Bernoulli shifts )Let $\varepsilon_{k}, k=0, \pm 1, \pm 2, \ldots$ be i.i.d. random variables that take the values 0 and 1 with probability $1 / 2$ each and let

$$
X_{n}=\sum_{k=1}^{\infty}(1 / 2)^{k+1} \varepsilon_{n-k}
$$

for $n=0,1,2, \ldots$. Then $\left\{X_{n}, n \geq 0\right\}$, is an ergodic stationary Markov chain taking values in $I=[0,1]$. The transition function is defined by $Q(x ;\{x / 2\})=1 / 2=$ $Q(x ;\{1+x\} / 2)$ for $x \in[0,1]$ and the stationary initial distribution is the restriction, $\lambda$ say, of Lebesgue measure to $I$. In this case, $L^{2}(\lambda)$ is a familiar space, and it is easy to relate the conditions in Theorem 2.4 to regularity properties of $g$. 
Proposition 3 in M. Maxwell and M. Woodroofe [7] (2000) showed that if $g \in L_{0}^{2}(\lambda)$ and

$$
\int_{0}^{1} \int_{0}^{1}[g(x)-g(y)]^{2} \frac{1}{|x-y|} \log ^{\delta}\left[\frac{1}{|x-y|}\right] d x d y<\infty
$$

for some $\delta>0$, then (2.12) holds. For example, if

$$
g(x)=\frac{1}{x^{\alpha}} \sin \left(\frac{1}{x}\right), \quad 0<x \leq 1,
$$

where $0<\alpha<1 / 2$, then (2.12) holds.

EXAMPLE 2. ( Lebesgue shifts )Let $U_{k}, k=0, \pm 1, \pm 2, \ldots$ be i.i.d. random variables that are uniformly distributed over $I=[0,1]$ and let

$$
X_{n}=\left(\cdots U_{n-2}, U_{n-1}, U_{n}\right), \quad n \geq 0 .
$$

Then $\left\{X_{n}, n \geq 0\right\}$, is a stationary Markov process taking values in $\mathcal{X}=I^{M}$, where $M$ denote the non-positive integers. The stationary initial distribution $\pi$ here is the countable product of copies of Lebesgue measure. Processes of the form $g\left(X_{n}\right)$, $n=0,1,2, \ldots$, include a wide class of stationary sequences.

Define measures $\Gamma_{\delta}^{1}$ on $\mathcal{X} \times \mathcal{X}$ by

$$
\Gamma_{\delta}^{1}\{B\}=\sum_{k=1}^{\infty} k^{\delta}\left(\pi \times \pi \times \lambda^{k}\right)\{(x, y, z):[(x, z),(y, z)] \in B\}
$$

for Borel set $B \subset \mathcal{X} \times \mathcal{X}$, where $x$ and $(x, z)$ denote the sequences $x=\left(\ldots, u_{-2}, u_{-1}, u_{0}\right)$ and $\left(\ldots, u_{-1}, u_{0}, z_{1}, \ldots, z_{k}\right)$ and $\lambda^{k}$ denotes Lebesgue measure on $I^{k}$.

M. Maxwell and M. Woodroofe [7] (2000) showed that if $g \in L_{0}^{2}(\pi)$ and

$$
\int_{\mathcal{X}} \int_{\mathcal{X}}[g(x)-g(y)]^{2} \Gamma_{\delta}^{1}\{d x, d y\}<\infty,
$$

for some $\delta>0$, then (2.12) holds. To illustrate the (3.2), observe that any $g \in L_{0}^{2}(\pi)$ may be written in the form

$$
g(x)=\sum_{k=0}^{\infty} g_{k}\left(u_{-k}, \ldots, u_{0}\right)
$$

where $x=\left(\ldots, u_{-2}, u_{-1}, u_{0}\right), g_{k}: \mathbb{R}^{k+1} \rightarrow \mathbb{R}$ are measurable, $\int_{\mathbb{R}} g_{k}\left(u_{-k}, \ldots, u_{0}\right) d u_{-k}=$ 0 for a.e. $\left(u_{-k+1}, \ldots, u_{0}\right), \sum_{k=1}^{\infty} \int g_{k}^{2} d \lambda^{k+1}<\infty$, and $\lambda^{k}$ denotes $k$-dimensional Lebesgue measure. Then (2.12) holds if for some $\delta>0$,

$$
\sum_{k=1}^{\infty} k^{1+\delta} \int g_{k}^{2} d \lambda^{k+1}<\infty
$$




\section{ACKNOWLEDGEMENTS}

The authors wish to thank Prof. L.M. Wu of Université Blaise Pascal and Prof Y.P. Zhang of Wuhan University for their helpful discussions and suggestions during writing this paper. And the authors thank Prof. Y. Derriennic and Prof. M. Lin for sending the key reference paper [3] to the authors.

\section{REFERENCES}

[1] R.N. Bhattacharya, On the functional central limit theorem and the law of the iterated logarithm for Markov processes. Z. Wahrs. verw. Gebiete., 60 (1982) 185201.

[2] Y.S. Chow and H. Teicher, Probability Theory. Sectond Edition. Springer-Verlag New York 1988.

[3] Y. Derriennic and M. Lin, Fractional Poisson equations and ergodic theorems for fractional coboundaries. Israel J. math. 123 (2001), 93-130.

[4] W. Feller, An introduction to probability theory and its applications. Second Edition. John Wiley and Sons, Inc. Vol 2, 1971.

[5] M.I. Gordin and B.A. Lifsic, The central limit theorem for stationary Markov processes. Dokl. Akad. Nauk SSSR, 19 (1978), 392-394.

[6] C. Kipnis and S.R.S. Varadhan, Central limit theorem for additive functionals of reversible Markov processes and applications to simple exclusions. Comm. Math. Phys.,104 (1986), 1-19.

[7] M. Maxwell and M. Woodroofe, Central limit theorems for additive functionals of Markov chains. Ann. Probab. 28(2) (2000), 713-724.

[8] S.P. Meyn and R.L. Tweedie, Markov chains and stochastic stability. SpringerVerlag New York 1993.

[9] R.P. Pakshirajan and M. Sreehari, The law of the iterated logarithm for a Markov process. Ann. Math. Statist. 41(3) (1970), 945-955.

[10] W.F. Stout, The Hartman-Wintner law of the iterated logarithm for martingales. Ann. Math. Statist. 41(6) (1970), 2158-2160.

[11] L.M. Wu, Forward-backward martingale decomposition and compactness results for additive functionais of stationary ergodic Markov processes. Annals de l'i.H.P., Série Probab and Stat., 35(2) (1999), 121-141.

Department of Mathematics and Statistics, Wuhan University, 430072 Hubei, China and College of Mathematics and Information Science, Henan Normal University, 453007 Henan, China.

E-mail address: yumiao728@yahoo.com.cn

Department of Mathematics and Statistics, Wuhan University, 430072 Hubei, China. E-mail address: study_yang@yahoo.com.cn 